\title{
How-To: Empirical IVIVR Without Intravenous Data
}

\author{
Aleksander Mendyk ${ }^{1, *}$, P aweł Konrad Tuszyński ${ }^{1}$, Mohammad Hassan Khalid ${ }^{1}$, Renata Jachowicz', \\ and Sebastian Polak ${ }^{2,3}$ \\ ${ }^{1}$ Department of Pharmaceutical Technology and Biopharmaceutics, Faculty of Pharmacy, Jagiellonian University - Medical College, \\ Medyczna 9 St., 30-688 Kraków, Poland \\ 2 Department of Pharmacoepidemiology and Pharmacoeconomics and Department of Social Pharmacy, Faculty of Pharmacy, Jagiellonian \\ University Medical College, Medyczna 9 Street, 30-688 Kraków, Poland \\ ${ }^{3}$ Simcyp (a Certara Company) Limited, Blades Enterprise Centre, John Street, Sheffield S2 4SU, UK
}

\begin{abstract}
IVIVR as an extension of IVIVC beyond the domain of linear modeling is a predictive model that binds the in vivo PK profile with the in vitro dissolution profile of a particular drug. Several computational intelligence-modeling tools for IVIVR were chosen and tested in this study: decision trees (randomForest), artificial neural networks (monmlp), genetic programming (rgp), and a recently published tool, RIVIVR. R statistical environment was used for numerical experiments. All of the above-mentioned tools succeeded in the creation of empirical relationships between in vivo and in vitro profiles without the need of the additional impulse curve (intravenous [iv] profile). The best results were found for genetic programming and decision trees. RIVIVR achieved a superior cost-effectiveness ratio, namely, short time of execution and high level of automation.
\end{abstract}

KEYWORDS: In vitro-in vivo relationship (IVIVR); empirical modeling; computational intelligence (CI); open source; R statistical environment.

\section{INTRODUCTION}

$\mathrm{B}^{\prime}$ y definition, an in vitro-in vivo correlation (IVIVC) is a predictive mathematical model that describes the relationship between the in vitro property of a dosage form and its in vivo response (1). It is an important tool for the development of dosage forms by making it possible to anticipate in vivo behavior of a drug based on cheap and reproducible in vitro assays. Dissolution testing as a surrogate of bioassay is attractive, and due to the substantial cost reduction, is a focus of the pharmaceutical industry and regulatory agencies. However, establishing IVIVC is still a challenge that not everyone attempts. According to Sharp (2), from 2009 to 2012, FDA received 36 applications (NDA and IND) with IVIVC established. Development of the most valuable point-to-point Level A IVIVC is not a trivial task as it requires three well-established key elements:

- Bioassay (in vivo data preferably including iv profile).

- Dissolution tests.

- Modeling.

Despite theFDAguidance introducing the conceptand development of IVIVC methodology (1), there is currently no complete and generic procedure that guarantees IVIVC establishment in all cases. Since dissolution testing and modeling are the most flexible elements of IVIVC, current trends for development of IVIVC methods mainly include biorelevant dissolution testing and sophisticated modeling techniques. As we represent the latter field, we would like to introduce our point of view demonstrating potential of

*Corresponding author. empirical modeling methods in IVIVR development, where IVIVR is understood as an extension of IVIVC beyond the domain of linear modeling (3). The aim of this work is to introduce currently available tools suitable for an empirical approach to establishing IVIVR.

\section{METHODOLOGY Modeling Tools and Approaches}

Modeling tools belonging to the computational intelligence area were chosen for this study: decision trees, artificial neural networks, genetic programming, and recently published tool RIVIVR. Due to its mature stage of development and wide recognition in the life sciences area, the R statistical environment (4) was chosen as our major platform for running the above-mentioned modeling systems.

Random Forest (RF) is a tree-based ensemble system, where many tree predictors are stacked together to form one model. Each tree is created on an independent and random sample taken from the training dataset. The generalization error of a forest depends on the errors of individual trees and the correlation among the trees (5). Random forests are suitable for classification problems, but they have been very efficient with regression and feature selection problems, too. For running this class of $\mathrm{Cl}$ algorithm, we used the randomForest module of $\mathrm{R}$ statistical environment (6).

Artificial neural networks (ANNs) are well-known modeling tools built in a manner similar to biological neural systems. A massive system of interconnections among 
units of ANNs together with well-established learning algorithms are sources of superb cognitive abilities of ANNs. The most common ANNs are so-called feed-forward ANNs with unidirectional signal processing and a supervised learning paradigm of work. For this class of tools, we used the $\mathrm{R}$ module monmlp (7), which from a practical point of view is characterized by highly reproducible results and relatively quick model development.

Genetic programming (GP) is a bioinspired algorithm based on evolution principles to solve complex problems. A high-level problem definition is solved into a creation of random solutions that are progressively refined through the process of variation and selection until a satisfactory solution is found. Artificial chromosomes are operational units undergoing several genetic operations like mutation, deletion, insertion, and crossing-over. The resulting solutions can be expressed in the form of mathematical equations, thus no more "black-box" problem is experienced when dealing with the final model. We found the R module rgp (8) very effective and used its socalled "symbolic regression" mode for models developed and described in this work.

RIVIVR is a new tool developed by Mendyk (9) for a direct, convolution-based, correlation of dissolution profiles with their pharmacokinetic counterparts. It is based on the optimization approach, where optimized parameters are points of a numerically developed iv time-concentration curve (in silico iv profile) used for convolution of the PK profile representing po administration of particular formulation. Once optimized on two or three available formulations, the in silico iv profile is used for convolutionbased prediction of a new formulation PK profile based on the new dissolution profile, thus accounting for the external validation.

The algorithm of RIVIVR execution is represented by following pseudo-code:

- gather in vitro and in vivo data-at least 2 formulations with different release rates

- step $=1$

- F step $=1$ THEN initialize artificial iv profile ELSE update artificial iv profile from p. 5

- step = step +1

- modify artificial iv profile

- convolve artificial iv profile with in vitro profile

- compare convolved PK profile with its corresponding observed counterpart

- compute prediction error

- IF error <= stop criterion THEN exit ELSE go back to p. 3

The above-mentioned procedure is realistic when using a strong optimization tool capable of global optimization with many adjustable parameters. R package GenSA (10), a general simulated annealing algorithm, works efficiently in this task and was therefore implemented as an integral part of RIVIVR. Default settings of RIVIVR include 100 points representing an artificial iv profile, which is initialized at the step no. 1 averaging in vivo profiles available as tutorial data. No information about the validation profile is included in this procedure.

\section{Data}

The data were extracted from the literature by careful selection of papers including both in vitro and in vivo data for at least three formulations with attempts to establish any form of IVIVC regardless of its level. To provide numerical representation of the profiles, graphs were scanned and processed by g3data software (11). Additionally, dissolution profiles subjected to model-based analysis, where several kinetic and empirical models were fitted and the parameters of the best-fitting models were added to the database to enhance the information gain of the dissolution data. In case of different drugs, input vector length could be different because of expected variations in fitting results. In other words, some models will be and some will not be included in certain cases. A threshold of normalized root-mean-squared error (NRMSE) (12) equal to $10 \%$ was applied to make the above distinction. Modelbased characterization of the dissolution profiles was performed using KinetDS software (13). Several models were used in this procedure: Weibull, Hill, KorsmeyerPeppas, Michaelis-Menten, Hixson-Crowell, Higuchi and zero- to third-order kinetics. Enhanced dissolution data sets were used for all the modeling tools described above except for the RIVIVR. Additionally, to synchronize the time scale of PK and dissolution profiles, a time-axis extension procedure was developed. Since in all cases the dissolution test protocol involved a shorter assay time than the bioassay, the time-axis extension procedure was performed as follows:

- Additional time point added to the in vitro dissolution profile reflecting last time point of the corresponding PK profile.

- $Q$ value (cumulative drug amount released) for the above time point set equal to the last $Q$ value of the original dissolution profile thus assuming no changes in the dissolution over the extended time.

- Resampling of both in vitro and in vivo curves with pchip routine of R package "signal" with a sampling step of 0.05 $\mathrm{h}$ to ensure overlapping time points in both profiles.

- Since RIVIVR is equipped with its own autonomous curve-sampling procedure, the above was performed for RIVIVR without final resampling of the profiles.

\section{Modeling Procedure}

The modeling approach was based on a simple assumption that it is possible to create a direct relationship between in vivo and in vitro data. Therefore, inputs for modeling tools were always in vitro data, whereas a PK profile was expected as the output (Figure 1). For each case of data identified in the literature, modeling included an estimation of internal and external validation error expressed as prediction error $\mathrm{PE}(\%)$ both for $C_{\max }$ and $A \cup C_{0-t}$ (1). Based on the dissolution kinetics, a standard 
approach was employed where formulations with fast (F) and slow drug release rates (S) were used as a model base, thus their PEs(\%) accounted for internal validation, whereas a moderate release rate (M) formulation was used for external validation. If there were more than three formulations available, then moderate-slow (MS), moderate-fast (MF), or both, formulations were introduced to the internal validation procedure. Computations were performed with use of an HPC cluster empowered with 340 threads and working under a Linux operating system (openSUSE) with self-written grid management software.

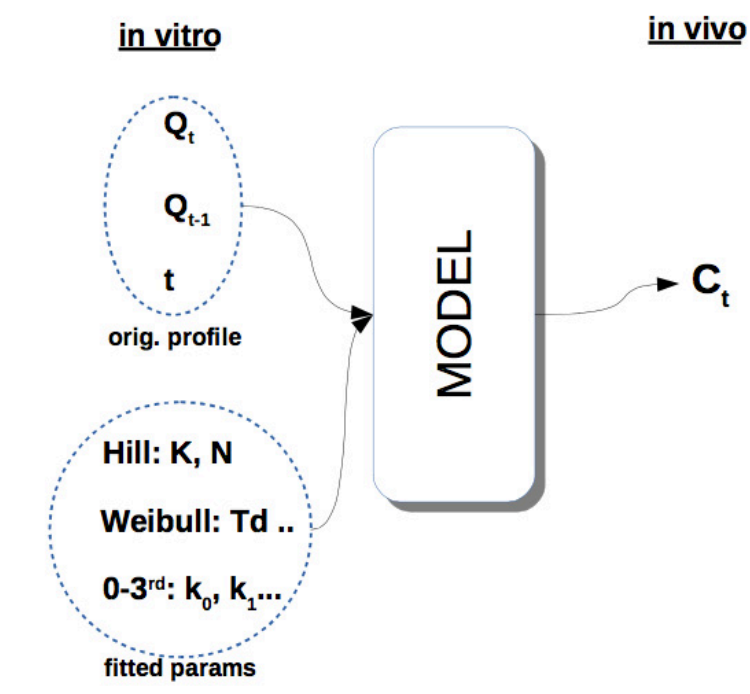

Figure 1. Experimental setup_-input and output data.

\section{RESULTS AND DISCUSSION}

After the search and selection phase, eight papers were chosen as a source of data for this study, containing dissolution and in vivo data for seven different model drugs: metformin (14), diltiazem $(15,16)$, metoprolol (17), ketoprofen (18), divalproex sodium (19), pramipexole (20), and oxycodone (21).

The modeling phase was carried out independently for each one of the tools described above and resulted in selection of the best generalizing models in each group, namely ANNs, RF, GP, and RIVIVR. A summary of external and internal validation of the best models in each class is included in Tables 1 and 2 with internal validation provided for GP and RIVIVR only. Our major focus on external validation was based on the assumption that to select the most suitable modeling tool, one needs the most challenging testing conditions. A brief analysis of the results leads to the conclusion that none of the tools completely succeeded in IVIVR development. Each tool failed at least twice in meeting the external validation $\mathrm{PE}(\%)$ criterion of $10 \%$ with GP exhibiting the highest success ratio of $75 \%$ (Table 1). In terms of absolute average $\mathrm{PE}(\%)$, the best predictability for $C_{\max }$ was observed for $\mathrm{RF}$, whereas RIVIVR was superior for $A \cup C_{0-t}$. In this ranking, GP was always in second position. Taking into account that GP results are mathematical formulas, GP might be regarded as a valuable tool for IVIVR development.

$$
C_{\text {in_vivo }}=\sqrt[8]{\operatorname{In}_{10}} \cdot \operatorname{In}_{11} \cdot \exp \left(-\frac{\mathrm{In}_{9}}{8}-C_{1}\right)
$$

where $C_{\text {in_vivo }}$ is the drug concentration in vivo, $\ln _{9}$ is the dissolution/PK profile time point, $\ln _{10}$ is $Q_{\mathrm{t}}, \mathrm{In}_{11}$ is $Q_{\mathrm{t}-1}$, and $C_{1}$ is the equation constant (1.3757).

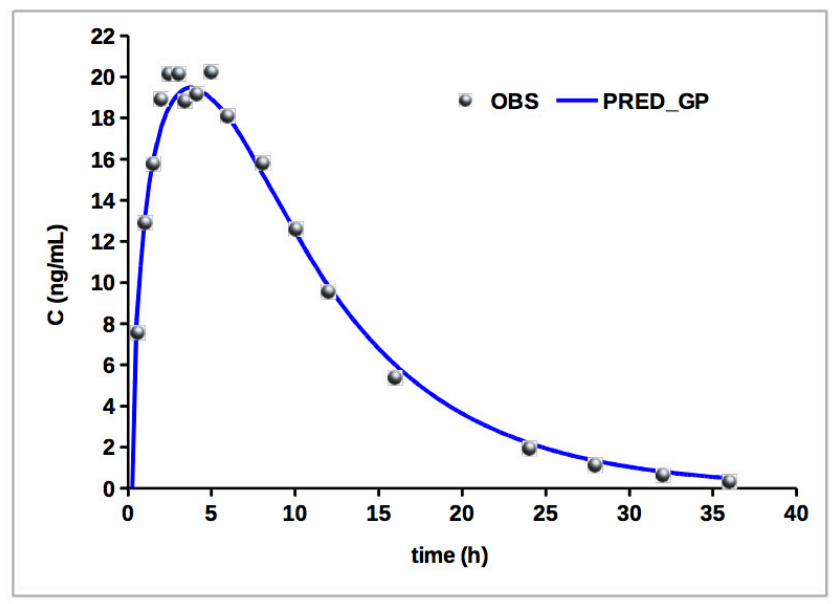

Figure 2. External validation for oxycodone dataset resulting from GP model (eq 1, PRED_GP).

The formula presented above shows one example of the many results generated after extensive experiments with the GP algorithm for the oxycodone dataset. The dataset itself and the model implemented in the R script are available from the "data/oxycodone" section of the supplementary materials (22). This equation looks simple enough and represents direct mapping of the dissolution profile into the PK profile without any additional information. In the course of its work, GP automatically reduced input variables from the original 11 inputs to the three-element vector. Selected crucial variables for this data set included only a time variable and a dissolution profile presented as the amounts of drug dissolved $(Q)$ in a specified time point $(t)$ and the previous one ( $t$ 1). It is an example of feature selection abilities of GP leading to the simplification of the final model. External validation for the oxycodone dataset obtained with this equation is presented in Figure 2. It clearly demonstrates good accordance between the simulated results and the observed ones. It is noteworthy that the oxycodone example was well represented by every tool used in this study. In case of the other data sets, complexity of the final models is substantially higher and therefore a full report of the discovered equations is presented in the supplementary materials (22). An interesting comparison of our results could be made with the ones obtained in the original source paper for diltiazem_1 data (15), where authors failed to meet predictability criteria for both internal and external validation using a deconvolution approach. On the contrary, although very complex (supplementary 
Table 1. External Validation as $\mathrm{PE}(\%)$ for $\mathrm{C}_{\max }$ and $\mathrm{AUC} \mathrm{C}_{0-t}$

\begin{tabular}{|c|c|c|c|c|c|c|c|c|}
\hline \multirow[t]{2}{*}{ Dataset } & \multicolumn{2}{|c|}{ ANNs } & \multicolumn{2}{|c|}{ Random Forest } & \multicolumn{2}{|c|}{ GP } & \multicolumn{2}{|c|}{ RIVIVR } \\
\hline & $C_{\max }$ & $A U C_{0-t}$ & $C_{\max }$ & $A U C_{0-t}$ & $C_{\max }$ & $A U C_{0-t}$ & $C_{\max }$ & $A U C_{0-t}$ \\
\hline metformin (13) & 40 & 29 & 9 & 10 & 18 & 18 & 11 & 11 \\
\hline diltiazem_1 (14) & 3 & 13 & 4 & 3 & 10 & 5 & 5 & 1 \\
\hline metoprolol (16) & 0 & 1 & 2 & 11 & 3 & 14 & 9 & 6 \\
\hline ketoprofen (17) & 4 & 0 & 6 & 9 & 7 & 1 & 0 & 8 \\
\hline diltiazem_2 (15) & 27 & 9 & 27 & 18 & 10 & 7 & 15 & 16 \\
\hline divalproex_s (18) & 15 & 4 & 1 & 3 & 2 & 4 & 29 & 4 \\
\hline pramipexole (19) & 5 & 2 & 12 & 5 & 15 & 6 & 9 & 4 \\
\hline oxycodone (20) & 2 & 3 & 7 & 0 & 4 & 1 & 1 & 1 \\
\hline Average & 12.0 & 7.6 & 8.5 & 7.4 & 8.6 & 7.0 & 9.9 & 6.4 \\
\hline FDA crit. & \multicolumn{2}{|c|}{$50.0 \%$} & \multicolumn{2}{|c|}{$62.5 \%$} & \multicolumn{2}{|c|}{$75.0 \%$} & \multicolumn{2}{|c|}{$62.5 \%$} \\
\hline
\end{tabular}

materials eq 2), the equation derived in our study resulted in an internal validation error below $15 \%$ (Table 2) and external validation error for moderate formulation as $10 \%$ for $C_{\max }$ and $5 \%$ for $A \cup C_{0-t}$ (Table 1). Yamashita et al. (23) also modeled with GP the data from the paper of Sirisuth et al. (15) and concluded suitability of GP for this task; however, they did not provide an estimation of external validation error or express their internal validation errors as $\mathrm{PE}(\%)$. The above findings point to the conclusion that GP is an efficient technique to find a predictable model, yet overall efficiency of this tool is substantially reduced by very long execution time. Development of an average model in this study took ca. two weeks of continuous work of a professional PC workstation based on Intel Xeon CPUs and capable of 24 threads parallel execution. This is a serious drawback of this technique, and there is a limited control over complexity/predictability ratio of the final models when using GP.

Table 2. Internal Validation for Best Modeling Tools

\begin{tabular}{|c|c|c|c|c|}
\hline \multirow{2}{*}{ Dataset } & \multicolumn{2}{|c|}{ GP } & \multicolumn{2}{|c|}{ RIVIVR } \\
\hline & $C_{\max }$ & $A U C_{0-t}$ & $C_{\max }$ & $A U C_{0-t}$ \\
\hline metformin (F) & 15 & 15 & 12 & 20 \\
\hline metformin (S) & 19 & 9 & 17 & 45 \\
\hline diltiazem_1 (F) & 15 & 0 & 4 & 6 \\
\hline diltiazem_1 (S) & 10 & 4 & 15 & 10 \\
\hline metoprolol (F) & 5 & 6 & 2 & 2 \\
\hline metoprolol (S) & 3 & 14 & 2 & 3 \\
\hline ketoprofen (F) & 8 & 5 & 1 & 0 \\
\hline ketoprofen (MS) & 16 & 13 & 9 & 1 \\
\hline ketoprofen (S) & 10 & 14 & 6 & 2 \\
\hline diltiazem_2(F) & 25 & 1 & 2 & 6 \\
\hline diltiazem_2(S) & 9 & 8 & 23 & 33 \\
\hline divalproex_s (F) & 10 & 10 & 1 & 5 \\
\hline divalproex_s (S) & 25 & 6 & 0 & 7 \\
\hline pramipexole (F) & 19 & 9 & 7 & 2 \\
\hline pramipexole (MF) & 3 & 1 & 4 & 1 \\
\hline pramipexole (S) & 1 & 5 & 10 & 7 \\
\hline oxycodone (F) & 8 & 8 & 6 & 2 \\
\hline oxycodone (S) & 6 & 8 & 2 & 3 \\
\hline
\end{tabular}

Errors expressed as $\mathrm{PE}(\%)$ for $C_{\max }$ and $A \cup C_{0-t}$
A
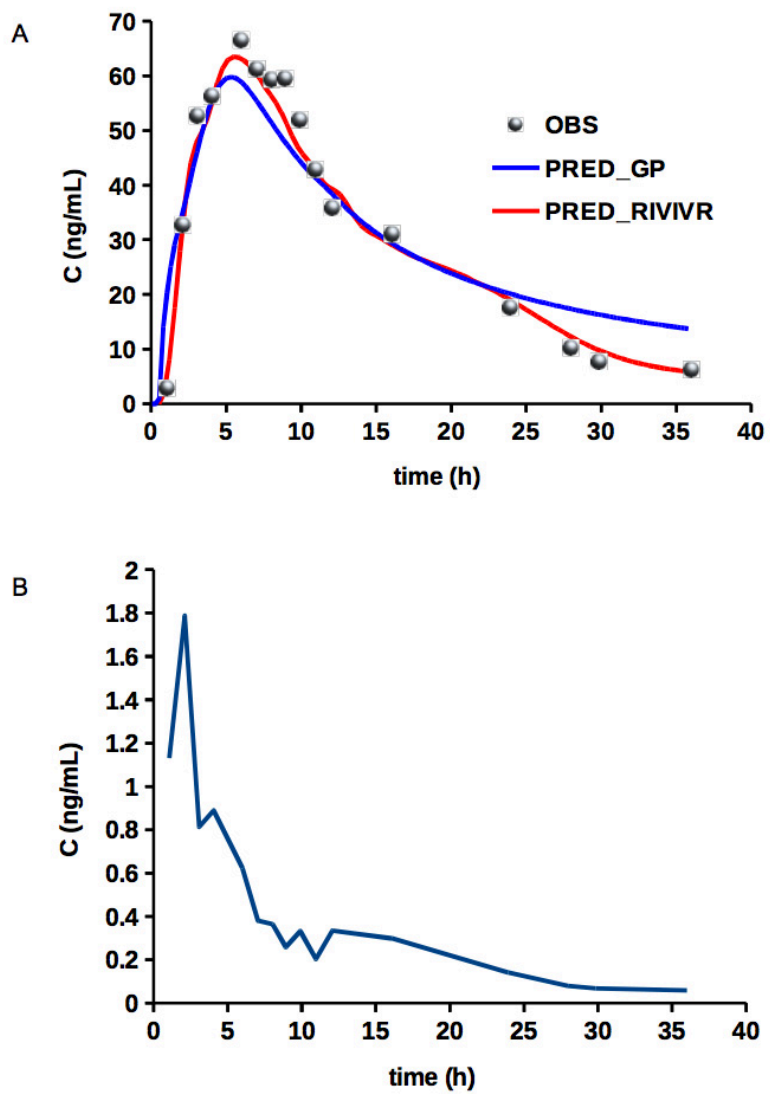

Figure 3. Results for diltiazem_1 dataset (OBS): (A) external validation for GP (PRED_GP) and RIVIVR (PRED_RIVIVR); (B) artificial iv profile generated by RIVIVR.

To overcome these obstacles, a new tool was developed and applied: RIVIVR. Regarding the above example of Sirisuth et al. (15), RIVIVR exhibited lower external validation errors than GP (5\% and $1 \%$ for $C_{\max }$ and $A \cup C_{0-t}$, respectively, Table 1 ) yet slightly higher internal validation errors reaching up to $15 \%$ for $C_{\max }$ (Table 2 ). In general, it could be concluded that predictability for both tools is comparable (Figure 3A), yet execution time favors RIVIVR as it approaches four minutes on a high-performance PC workstation like the one used for GP execution for about 
two weeks. It is a clear indication that a major advantage of RIVIVR is its ability to map dissolution data directly with PK profiles using the profiles as the only input information and executing on an average PC or laptop. Therefore, it could be used for purely empirical reasoning by a trial-anderror approach just to check if this simplistic convolutionbased approach is feasible in a particular case. Although RIVIVR does not present its results as a mathematical formula, it generates an artificial iv profile used in the convolution procedure to derive po profiles. This iv curve is also reported and presented as an output, thus it can be used with other modeling approaches providing means for RIVIVR validation (Figure 3B).

RIVIVR shares some code with and uses the "NumConv" routine from Rivivc, which is an official $R$ module for IVIVC developed in our lab (9). Because of that, RIVIVR does not require sampling points of all the curves to be the same since they are automatically adjusted by the software itself with a user-controlled accuracy level. Moreover, it is not relevant if the dissolution data are presented as percentages or in the range of $0-1$, as RIVIVR does not use any mechanistic assumptions and the resulting artificial iv curve reflects the dissolution data range elegantly. Based on the presented reasoning, it has to be acknowledged that the artificial iv curve derived numerically by RIVIVR (Figure 3) has no physical meaning and cannot be interpreted in such a way. In this approach, it is more of a scaling factor than the concentration-time profile.

The above reasoning did not include RF or ANNs. For the latter, its poor predictability is an explanation of this exclusion, yet RF excelled in terms of predictability of $C_{\max }$. The reason for our focus on GP and RIVIVR is the transparency of these tools, which allows full insight into their mode of work and way of data handling. This is certainly an advantage in terms of industrial applications due to the need for full validation of the models. On the contrary, ANNs are classical examples of "black-box" models that are impossible to reveal their internal way of data processing. In theory, the RF mode of work should be traceable as a set of choices among branches and nodes of decision trees. However, RF is an ensemble system containing several hundreds of decision trees in a single model. This poses a serious problem with any approach to disclose internal information flow and complete paths of decision-making performed by the system to provide its final answer corresponding to the data presented at the input. For the data used in this study, the largest RF models contained 1,000 trees, each one built on a maximum number of 600 nodes. These numbers provide a clear perspective on the complexity of RF models. The final point is the execution time and required computational resources. In this work for ANNs, we needed roughly three days of work of the Xeon-based 24-threads PC workstation cited above, whereas for RF it decreased to $4 \mathrm{~h}$. Still these models cannot be developed efficiently on a regular PC or a laptop.

\section{CONCLUSIONS}

We have demonstrated that empirical models can be used for direct mapping of dissolution profiles to PK profiles as confirmed by other authors (23). Such an IVIVR approach is especially applicable where there are no results available from iv administration as required by classical deconvolution/convolution methods. However, this is a still a high-risk approach as among successful correlations, our results included several failures in meeting FDA validation criteria. Moreover, classical $\mathrm{Cl}$ tools like ANNs or Random Forest suffer from a commonly known problem of being "black boxes" and are not good subjects for industrial applications due to their inability of full validation of the model. Regarding the above, we provided ready-to-use solutions, where no more hidden relationships are present. A simple mathematical formula representing an IVIVR model is a good alternative to any other approach, yet its development from scratch is not a trivial task. We found the R module rgp and its mode of running called "symbolic regression" apt to this task and successful in terms of predictability. However, the cost of model development in terms of required computational power and execution time prevents us from using it as a primary tool. Thus, we developed RIVIVR software that employs a classical convolution approach and optimization of artificial, created numerical curves - the latter representing impulse, namely iv administration PK profile. RIVIVR, though moderately precise, can be a valuable first-choice tool for empirical IVIVR owing to its short execution time and simplicity of design.

In the future, further development is envisaged for RIVIVR that includes:

- Code optimization for speed improvement.

- Improvement of stability and predictive power of models by introduction of carefully limited noise in the tutorial data.

- Graphical user interface (GUI).

We provide all of the above-mentioned tools as ready-to-use R scripts available under GNU GPL license via sourceforge.net webpage:

- GP (24)

- RF (25)

- ANNs (26)

- $\operatorname{RIVIVR}(27)$

The above software is to be used AS IS without any warranty or liability, yet at no cost and free to use both personally and commercially. We believe that this contribution will improve development of good quality IVIVC and IVIVR models.

\section{SUPPLEMENTARY MATERIALS}

To make it easier to follow our modeling strategies and to facilitate readers' own experiments, we published Supplementary materials (22) through the sourceforge. net server. Supplementary materials contain the following elements: 
- A full report of GP modeling for all datasets accompanied with a short description of the "data" section (SupplementaryMaterials_Equations.pdf) is in the root directory.

- All datasets for developing equations with GP (learningSet.txt, testingSet.txt) and 10-fold crossvalidation procedure are in the "data" directory and its subdirectories named after datasets presented in Table 1.

- Standard subdirectories "GP_modeling" and "optimization"-the former with R scripts for GP modeling and the latter with $R$ scripts for testing internal and external validation of the equations developed with $\mathrm{GP}$-are found in the subdirectories of "data" directory.

\section{ACKNOWLEDGMENTS}

One of the authors (Mohammad Hassan Khalid) is supported by the IPROCOM Marie Curie Initial Training Network, funded through the People Programme (Marie Curie Actions) of the European Union's Seventh Framework Programme FP7/2007-2013/ under REA grant agreement No. 316555.

\section{CONFLICT OF INTEREST}

No conflict of interest has been declared by the authors.

\section{REFERENCES}

1. Extended Release Oral Dosage Forms: Development, Evaluation, and Application of In Vitro/In Vivo Correlations; Guidance for Industry; U.S. Department of Health and Human Services, Food and Drug Administration, Center for Drug Evaluation and Research (CDER), U.S. Government Printing Office: Washington, DC, 1997. http://www.fda.gov/downloads/drugs/ guidancecomplianceregulatoryinformation/ guidances/ucm070239.pdf (accessed April 16, 2015).

2. Sharp, S. S. FDA's Experience on IVIVC-New Drug Products. Presented at PQRI Workshop on Application of IVIVC in Formulation Development, Bethesda, MD, September 5-6, 2012. http://www.researchgate.net/ publictopics.PublicPostFileLoader.html?id=5442346a d039b1d55c8b45e5\&key=1c3a11a1-52c2-4094-bd1a2a2d4b85a3f0 (accessed April 16, 2015).

3. Polli, J. E. IVIVR versus IVIVC. Dissolution Technol. 2000, 7 (3), 6-16.

4. The R Project for Statistical Computing. R Foundation Web site. http://www.R-project.org (accessed April 16, 2015).

5. Breiman, L. Random forests. Mach. Learn. 2001, 45 (1), 5-32. DOI: 10.1023/A:1010933404324.

6. Liaw, A. randomForest: Breiman and Cutler's random forests for classification and regression. http://cran.rproject.org/web/packages/randomForest/index.html (accessed April 16, 2015).

7. Cannon, A. J. monmlp: Monotone multi-layer perceptron neural network. http://cran.r-project.org/ web/packages/monmlp/index.html (accessed April 16, 2015).

8. Flasch, O.; Mersmann, O.; Bartz-Beielstein, T.; Stork, J.; Zaefferer, M. rgp: R genetic programming framework. http://cran.r-project.org/web/packages/rgp/index. html (accessed April 16, 2015).

9. Mendyk, A.; Polak, S. Rivivc: In vitro in vivo correlation linear level A software. http://cran.r-project.org/web/ packages/Rivivc/index.html (accessed April 16, 2015).

10. Gubian, S.; Xiang, Y.; Suomela, B.; Hoeng, J. GenSA: Functions for Generalized Simulated Annealing. http:// cran.r-project.org/web/packages/GenSA/index.html. (accessed April 16, 2015).

11. Frantz, J. g3data Software. http://www.frantz.fi/ software/g3data.php (accessed April 16, 2015).

12. Pacławski, A.; Szlęk, J.; Lau, R.; Jachowicz, R.; Mendyk, A. Empirical modeling of the fine particle fraction for carrier-based pulmonary delivery formulations. Int. J. Nanomed. 2015, 10, 801-810. DOI:10.2147/ijn.s75758.

13. Mendyk, A.; Jachowicz, R.; Fijorek, K.; Dorożyński, P.; Kulinowski, P.; Polak, S. KinetDS: An Open Source Software for Dissolution Test Data Analysis. Dissolution Technol. 2012, 19 (1), 6-11. DOI: 10.14227/DT190112P6.

14. Balan, G.; Timmins, P.; Greene, D.; Marathe, P. In VitroIn Vivo (IVIVC) Correlation Models for Metformin after Administration of Modified-Release (MR) Oral Dosage Forms to Healthy Human Volunteers. J. Pharm. Sci. 2001, 90 (8), 1176-1185. DOI: 10.1002/jps.1071.

15. Sirisuth, N.; Augsburger, L. L.; Eddington, N. D. Development and Validation of a Non-Linear IVIVC Model for a Diltiazem Extended Release Formulation. Biopharm. Drug Dispos. 2002, 23 (1), 1-8. DOI: 10.1002/ bdd.270.

16. Korhonen, O.; Kanerva, H.; Vidgren, M.; Urtti, A.; Ketolainen, J. Evaluation of novel starch acetatediltiazem controlled release tablets in healthy human volunteers. J. Controlled Release 2004, 95 (3), 515-520. DOI: 10.1016/J.JCONREL.2003.12.026.

17. Sirisuth, N.; Eddington, N. D. The influence of first pass metabolism on the development and validation of an IVIVC for metoprolol extended release tablets. Eur. J. Pharm. Biopharm. 2002, 53 (3), 301-309. DOI: 10.1016/ S0939-6411(01)00248-X.

18. Corrigan, O. I.; Devlin,Y.; Butler, J. Influence of dissolution medium buffer composition on ketoprofen release from ER products and in vitro-in vivo correlation. Int. J. Pharm. 2003, 254 (2), 147-154. DOI: 10.1016/S03785173(03)00004-8.

19. Dutta, S.; Qiu, Y.; Samara, E.; Cao, G.; Granneman, G. R. Once-a-Day Extended-Release Dosage Form of Divalproex Sodium III: Development and Validation of a Level A In Vitro-In Vivo Correlation (IVIVC). J. Pharm. Sci. 2005, 94 (9), 1949-1956. DOI: 10.1002/jps.20387.

20. Soto, E.; Haertter, S.; Koenen-Bergmann, M.; Staab, A.; Trocóniz, I. F. Population In Vitro-In Vivo Correlation Model for Pramipexole Slow-Release Oral Formulations. 
Pharm. Res. 2010, 27 (2), 340-349.

21. Mundin, G. E.; Smith, K. J.; Mysicka, J.; Heun, G.; Krämer, M.; Hahn, U.; Leuner, C. Validated in vitro/ in vivo correlation of prolonged-release oxycodone/ naloxone with differing dissolution rates in relation to gastrointestinal transit times. Expert Opin. Drug Metab. Toxicol. 2012, 8 (12), 1495-1503. DOI: 10.1517/17425255.2012.729578.

22. Mendyk, A.; Tuszyński, P. K.; Khalid, M. H.; Jachowicz, R.; Polak, S. R scripts for multivariate analysis supplementary materials. Sourceforge Web site. http:// sourceforge.net/projects/rscriptsmultivariate/files/ RIVIVR/supplem_materials (accessed April 16, 2015).

23. Yamashita, F.; Fujita, A.; Zhang, X.; Sasa, Y.; Mihara, K.; Hashida, M. Computer-based Evolutionary Search for a Nonlinear Conversion Function for Establishing In Vitro-In Vivo Correlation (IVIVC) of Oral Drug Formulations. Drug Metab. Pharmacokinet. 2012, 27 (3), 280-285. DOI: 10.2133/dmpk.DMPK-11-RG-075.

24. Pacławski, A.; Mendyk, A.; Szlęk, J. A script for running genetic programming with rgp module. http:// sourceforge.net/projects/rscriptsmultivariate/files/ rgp/ (accessed April 16, 2015).

25. Mendyk A.; Pacławski A.; Szlęk J. A script for running Random Forest with $\mathrm{R}$ randomForest module. http:// sourceforge.net/projects/rscriptsmultivariate/files/ randomForest/ (accessed April 16, 2015).

26. Mendyk, A.; Szlęk, J.; Pacławski, A. A script for running feedforward ANNs with R monmlp module. http:// sourceforge.net/projects/rscriptsmultivariate/files/ monmlp/ (accessed April 16, 2015).

27. Mendyk, A. R scripts for multivariate analysis. RIVIVR software. http://sourceforge.net/projects/ rscriptsmultivariate/files/RIVIVR (accessed April 16, 2015). 